\title{
PERSONAL HEALTHCARE SYSTEM BASED ON ARTIFICIAL INTELLIGENCE
}

\section{${ }^{*}$ Bahaa S. Mostafa ${ }^{1}$}

Abbas Hussain miry ${ }^{2}$
Tariq M. Salman ${ }^{3}$

1) MSc. Student, Electrical Engineering Department, Al-mustansiriyah University, Najaf, Iraq

2) Asst. Prof. Dr. Electrical Engineering Department, Al-mustansiriyah University, Baghdad, Iraq

3) Asst. Prof. Dr. Electrical Engineering Department, Al-mustansiriyah University, Baghdad, Iraq

\begin{abstract}
With the increasing population in the world, especially the elderly, there is an urgent need to provide appropriate medical care for them. Therefore, a personal health monitoring system was proposed and implemented, and the results analyzed by artificial intelligence using fuzzy logic. Fuzzy logic has proven to be an effective tool for building smart decision-making systems based on the knowledge and observation of health care practitioners. An Atmega controller was used to achieve the proposed work function, as well as a heart rate pulse sensor and an infrared temperature sensor were Chosen, to measures skin and room temperatures. Fuzzy logic through inputs gives the person a condition, whether it is normal, abnormal, or in danger (degree of risk). Our model is expected to be effective in terms of accuracy, speed, low cost and smarter towards results compared to other prevailing traditional methods. Finally, the proposed system can be developed to make it able to predict the diseases that may happen to a person and also send an alert in the event of a danger.
\end{abstract}

Keywords: Atmega microcontroller board; fuzzy logic (FL); Pulse rate; Temperature sensor.

\section{Introduction}

The demand for home health care is influenced by many factors, including demographic trends; the needs of home health care patients; and improvements in health care to more costeffective solutions, such as managed care and other risk-sharing schemes. Chronic heart disease patients in stable condition belonging to one of four specific risk groups: arterial hypertension, malignant arrhythmias, heart failure and post-infarction rehabilitation compulsory cardiac disease patients require continuous monitoring of their health. One way of offering home healthcare is to use interactive technologies, such as providing emergency services and home health monitoring['].

Fuzzy logic-based algorithms also demonstrated potential to increase the efficiency of health care professionals by imitating processes of human thought in complex circumstances and performing common, repetitive tasks that are unfit for humans. In practice, a specific model for a medical condition may not exist, or modeling may be too difficult; in such cases, fuzzy logic is considered an appropriate approach, as human minds work from approximate data, extract concrete information, and build crisp solutions. 
FL has shown itself capable of moving human and expert information to computer-manageable models using linguistic words, such as that of a doctor. Fuzzy logic inference systems \{FIS utilize a set of if-then-rules based on clinical experience (physicians in the case of this work) to help, for example, the diagnosis of medical problems or the suggestion of appropriate care for a specific condition $[2,3]$.

The major benefits of fuzzy logic in health-care organizations are improved outcomes of treatment, reduced costs, enhanced patient care, better risk control, better disease detection, and reduced errors. The key feature of the health care system is checking vital signs of the patient, such as skin temperature, Electrocardiography (ECG), respiration, percentage of oxygen saturation, blood pressure, motion and heart rate .

On the subject of personal healthcare, several studies have been proposed, analyzed, and investigated(related work), for example, Healthcare Monitoring System Based On Pulse Sensor[4],Simulation, Feature Extraction And Disorder Detection(using Fuzzy Logic)Of Uterine Contractions[5], Simulation Models Of Emergency Department In Hospital [6], Development of Decision Support System for the Diagnosis of Arthritis Pain for Rheumatic Fever Patients: Based on the Fuzzy Approach[7], A fuzzy logic-based warning system for patients classification [8].

Each study in the previous works has a different type of machine learning used, a different number of sensors, different types of vital signs, different microcontrollers used, different ages of patient.

In this paper, the skin temperature, room temperature, heartbeat rate is measured, then analyze it by using fuzzy logic with many rules to decide the degree of risk, and display the results of measurements and decision. The microcontroller that uses in this paper is Atmega microcontroller .

In the future, the proposed system can lead to the development of a comprehensive healthcare monitoring system in the hospital unit or department. This project would also support ICU patients, and it can also facilitate the monitoring of the elderly in nursing homes.

The organization of this paper is as follows: Sec. 2 introduces fuzzy logic, Sec.3contains the designed system, theoretical operation concepts, and implementation. In Sec. 4, the results of the developed system are presented, demonstrated, and discussed. Finally, the conclusion of the paper is presented in Sec. 5 .

\section{Fuzzy logic}

Fuzzy logic theory was introduced in 1960's, and is built on the theory of fuzzy sets. In fuzzy sets, membership of an element would be not only false or true as in Boolean logic, and it is not smoother but more incremental. In fuzzy logic the degree of membership of an element in the interval $[0,1]$ can be any real number. Fuzzy logic has the advantage of helping us to overcome the ambiguity and inaccuracy of many real-world problems. Essentially it simulates human reasoning and decision-making abilities, based on less precise intelligence. The Membership function is a graphic display of the degree to which an input is involved. It assigns a weight to each of the processed inputs, recognizes functional overlap between inputs and eventually decides on an output response. Readers are directed to the study of Ross and 
Zadeh for a thorough description of the theory behind the FL. $[9,10]$

A method is required to define complex systems accurately, so that they can be approximated with a rational and trackable model. Fuzzy logic is a multi-valued system of logic which fulfills that function. Using FL needs four components: fuzzification, an inference, a fuzzy rule base, and defuzzing. One of Fuzzy logic's basic concepts is the degree of membership calculated by the use of the fuzzy input collection to "fuzzify" data point. The device designer decides the input fuzzy set to decompose into membership functions the entire range of potential input values. Each membership function has either a value of 1 or 0 , the maximum and minimum input value. Various types of membership may be used, including triangular, Gaussian, and trapezoidal forms. The most popular and easiest to understand are triangular and trapezoidal membership features, which can be fused into a fluid setting by achieving the minimum input value of each function to the center point of the prior one [11,12].

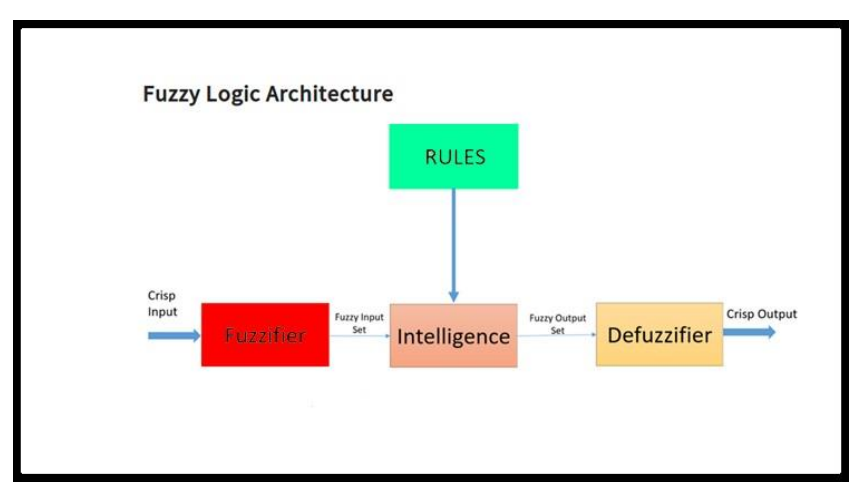

Figure 1. Fuzzy Logic Architecture

Fuzzy inference is the process of formulating the mapping from a given input to an output using fuzzy logic. The mapping then provides a basis for making decisions, or for identifying patterns. The inference method of Fuzzy involves all the sections specified in membership functions, logical operations and if-then legislation. The fuzzy inference method is composed of five of the following:

* Fuzzification of variables data

* Blurry operator function (AND or OR) in the precedent

* Engagement from the preceding to the consequent

* Analysis of the effects in the rules

* Defuzzification

Fuzzy reasoning is also well suited for clinical control systems, since the benefit from the results often have a wide variety that can lead to several diagnoses.

\section{System Design, theoretical concepts and Implementation}

\subsection{System Architecture}

The designed system architecture for the proposed Personal healthcare monitoring system using Fuzzy logic as shown in Fig 1. Room temperature sensor, skin temperature sensor and heart rate sensor will be putted on and near the patient. With the aid of the sensors, healthcare condition for the patients can be observed and then the information of these sensors' records can display on laptop, LCD, etc.

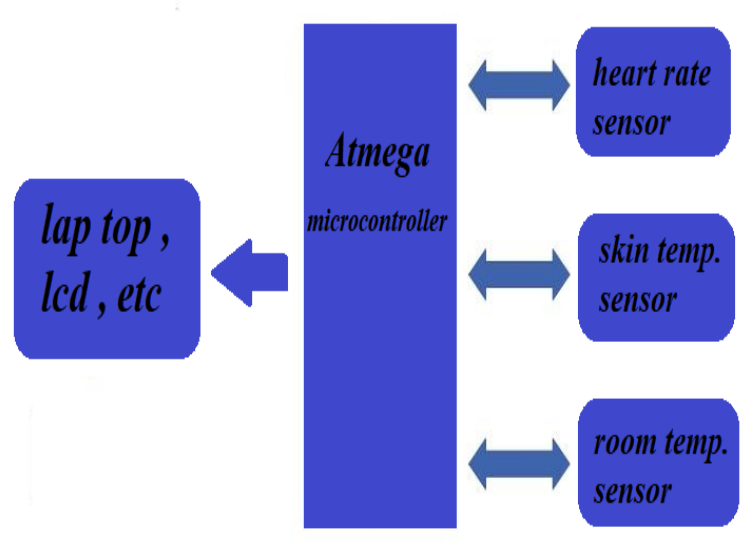

Figure 2. System architecture 


\subsection{Fuzzy logic basics}

The Fuzzy logic (FL) approach have utilized. The (FL)-based methodology focuses on the decision-making purpose. It is mainly used to get deficient data to take decision with the concepts called true or false and degrees of truth. The fuzzy set fully include the classical set. The membership function property is utilized for implementing the fuzziness of elements in the set that will have the solution based on the experiment in spite of knowledge. The weighted rate methodology is used to implement the membership function inside the fuzzy interference system[13-15].

In our work, the collected data form the sensors is analyzed by fuzzy logic. The graphs based on the inferences made using fuzzy logic are generated. The advantage of using fuzzy logic are that we do not require many data sets to analyze the newly data collected. Another advantage of Fuzzy logic is its power of interpretability and simplicity [16]. Mamdani Inference method is used as a Fuzzy methodology that is a commonly used methodology and simplest method because its structure depending on min-max operations. The Mamdani fuzzy inference method have also chosen because of its wide spread acceptance and it is well suited for human inputs. The output from the Mamdani method can also be sufficiently transferred to a linguistic form[14].

The fuzzy rule base system is utilized to produce the outputs according to the offered input for the system. In this paper, 2 input parameters will be entered to the system. The first (skin temperature) consist of 3 membership functions and the second (heart rate) consist of 4 membership functions. The output (degree of risk) consist of 5 membership functions. The number of rules is calculated based on each given parameter's membership function. The whole number of rules framed is 12 as shown in (Table 1). The membership function values and the fuzzy rules are introduced by researcher's proposition based on the fuzzy inference concept.
Table 1. The fuzzy rule table with different categories of the input and output parameters

\begin{tabular}{|c|c|c|c|}
\hline & \multicolumn{2}{|c|}{ Input } & Output \\
\hline & Skin temperature & Heart pulse rate & Degree of risk \\
\hline 1 & Moderate & Normal & No \\
\hline 2 & Hypo thermal & Normal & Low \\
\hline 3 & Hyper thermal & Normal & Low \\
\hline 4 & Moderate & Tachycardia & Med \\
\hline 5 & Hypo thermal & Tachycardia & High \\
\hline 6 & Hyper thermal & Tachycardia & High \\
\hline 7 & Moderate & Bradycardia & High \\
\hline 8 & Moderate & Severe tachycardia & High \\
\hline 9 & Hyper thermal & Bradycardia & V. high \\
\hline 10 & Hypo thermal & Bradycardia & V. high \\
\hline 11 & Hypo thermal & Severe tachycardia & V. high \\
\hline 12 & Hyper thermal & Severe tachycardia & V. high \\
\hline
\end{tabular}

Figure. 2 demonstrates the Fuzzy Inference System in which the heart pulse rate and skin temperature are the input values. After finish Fuzzification, Defuzzification methodology is used for producing the output for degree of risk.

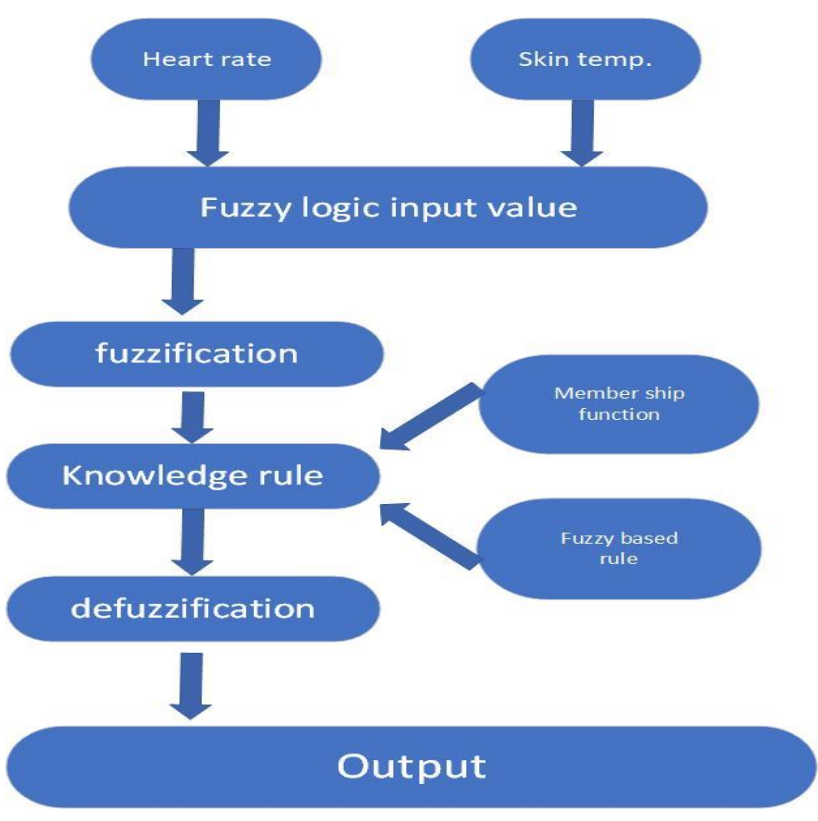

Figure 3. Fuzzy Inference System

The input membership functions are formulated using the trapezoidal function, and the output membership function is formulated using the triangle membership function.

Fig. 3 demonstrates the membership function for heart rate whose parameters for analyzing heart 
rate are bradycardia, normal, tachycardia and severe tachycardia [17].

Fig. 4 show the membership function for skin temperature whose parameters for analyzing skin temperature are hypo, moderate and hyper.

Fig. 5 show the membership function for output whose parameters for analyzing output are no, low, medium, high and very high.

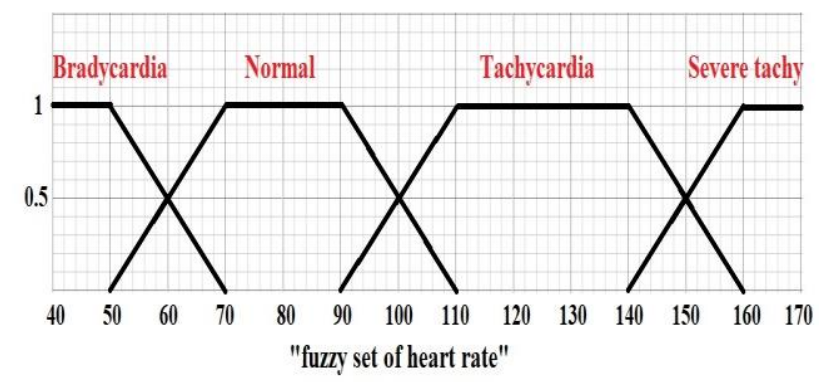

Figure 4. membership function for heart rate

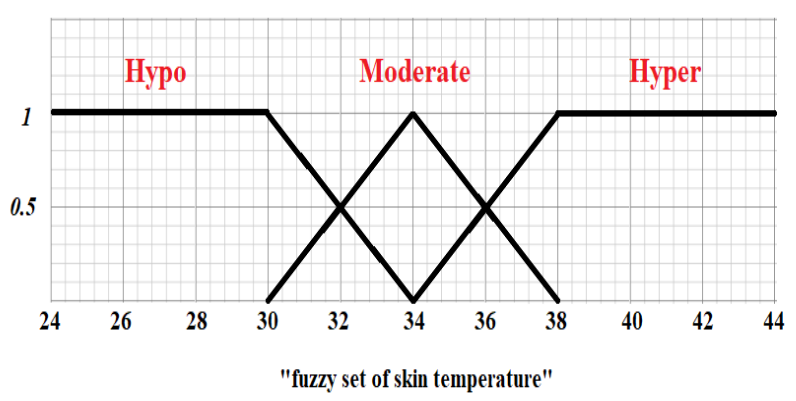

Figure 5. membership function for skin temperature

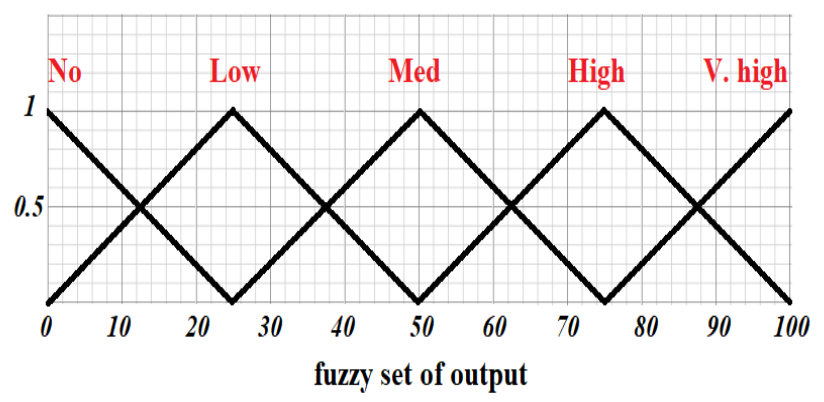

Figure 6. membership function for output

\subsubsection{Arduino mega}

The Arduino Mega 2560 is a board based on the ATmega2560 (datasheet) Microcontroller. This Arduino board has 54 digital input / output pins (of which 14 can be used as PWM outputs), 16 analog inputs, a $16 \mathrm{MHz}$ crystal oscillator, 4 UARTs (serial hardware ports), a USB link, a powerful jack and an ICSP header, and a reset button. It contains everything required to support the microcontroller; simply link it to a device with a USB cable, or power it to get going with an AC to-DC adapter or battery. The Arduino can be operated by an external power supply or by USB link. External power can come from either an AC-to - DC converter, or a battery [18-19].

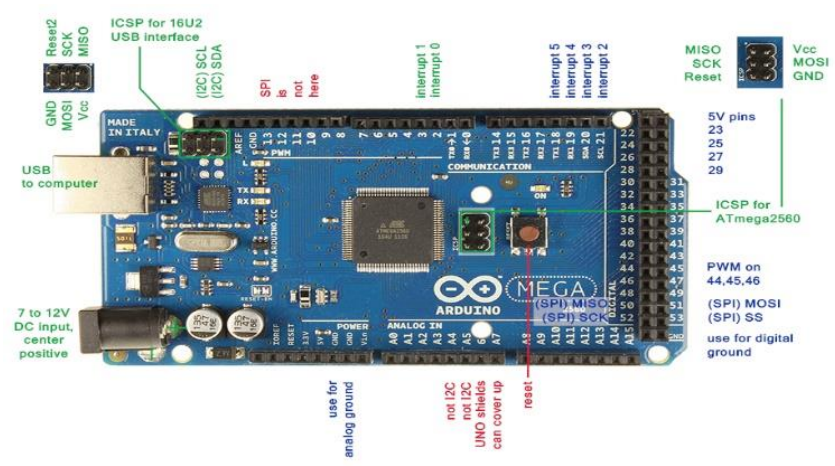

Figure 7. Arduino mega

\subsubsection{Temperature IR Sensor}

The MLX90614 is an Infra-Red Thermometer which is non-contact temperature monitoring. The MLX90614 integrates a low-noise amplifier, 17-bit ADC and an effective DSP package while achieving high thermometer accuracy and resolution.

The thermometer comes in a factory fitted with a digital SM Bus output that gives full access to the temperature measured in the $0.02{ }^{\circ} \mathrm{C}$ maximum temperature range(s) [20].

\subsection{Implementation}




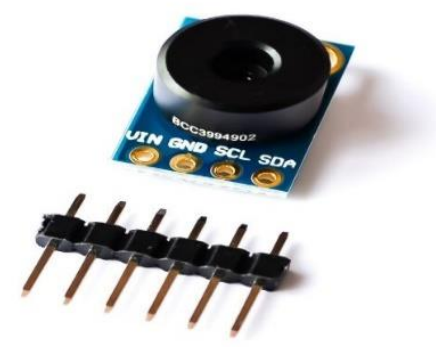

Figure 8. Temperature IR Sensor

\subsubsection{Grove - Ear-clip Heart Rate Sensor}

The heart rate ear hook set includes an ear clip and a receiver. The heart rate calculator can be used to track the patient and sports heart rate. The device has a good industry, so its readings are very accurate, and its energy consumption is low and very portable

To ensure the accuracy of the results to measure the heart rate in one minute, we made the program measure the pulses for 15 seconds and then multiply the number by 4 , so we see in the results a delay period between one result and another between (15 to 20) seconds [21].

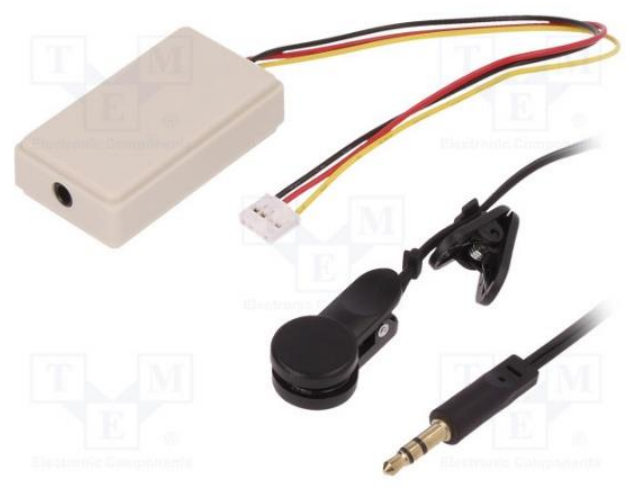

Figure 9. Ear-clip Heart Rate Sensor

The following figure show the installation of the Atmega microcontroller board with sensor, and also shows how to supply the sensors connected to the power $(5 \mathrm{v})$ by red wire and ground by black wire through mini board. Where the information transfer wire of the heartbeat sensor was connected to one of the analog blocks and the information wire of the skin temperature sensor was connected by red wire to the two blocks, Or Serial Clock (or SCL) and Serial Data (or SDA) lines are. The power of the microcontroller can be supplied in several ways, the first by laptop from the USB port and the second by an external adapter that connects to its power jack in the controller and the third by batteries that connect to the port (Vin).

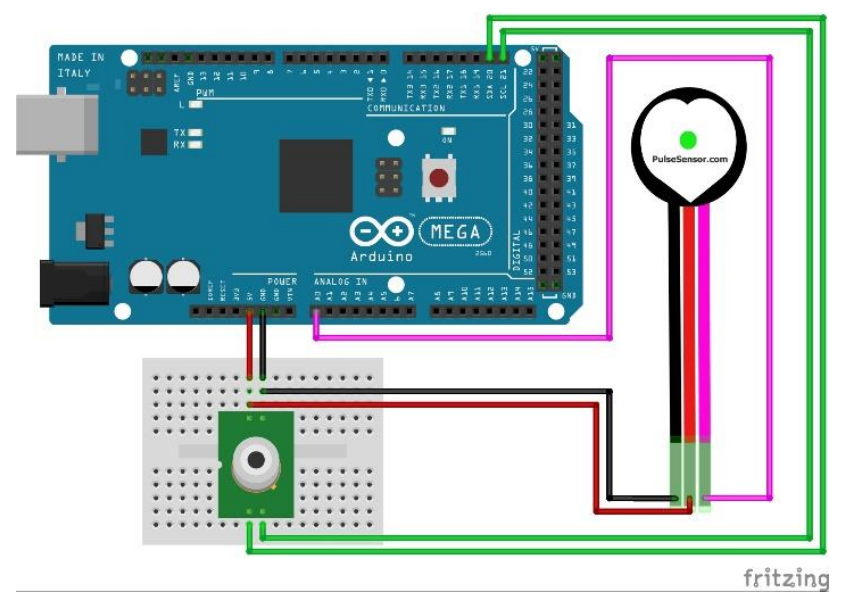

Figure 10. A schematic diagram of circuit

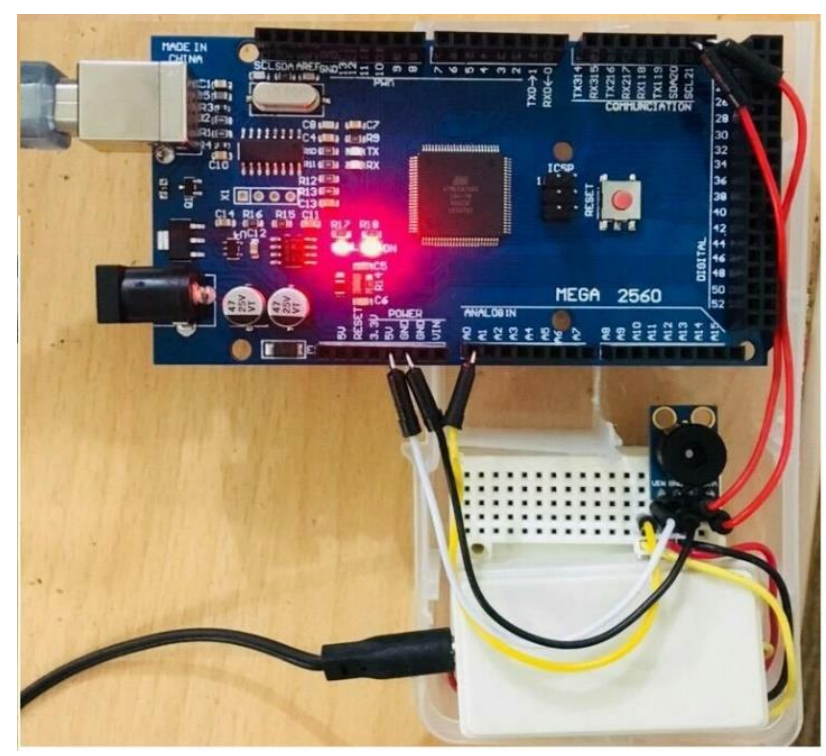

Figure 11. User prototype 


\section{Results And Discussion}

\subsection{Operating Mechanism}

The department's connections to the installation and testing phase of the smart health metering system are shown as in the following figure after mentioning the steps of installing the device.

STEP.1: The Pulse sensor is set at the patient's ear. This involves an IR-sensor. Get pulse from the sensor in every heartbeat. This sensor output from the signal conditioning unit for amplification is provided to the Atmega microcontroller.

STEP 2: The patient places his finger over the opening underneath the skin temperature sensor, so the sensor takes the value of the skin temperature through the IR and then goes to the Arduino mega.

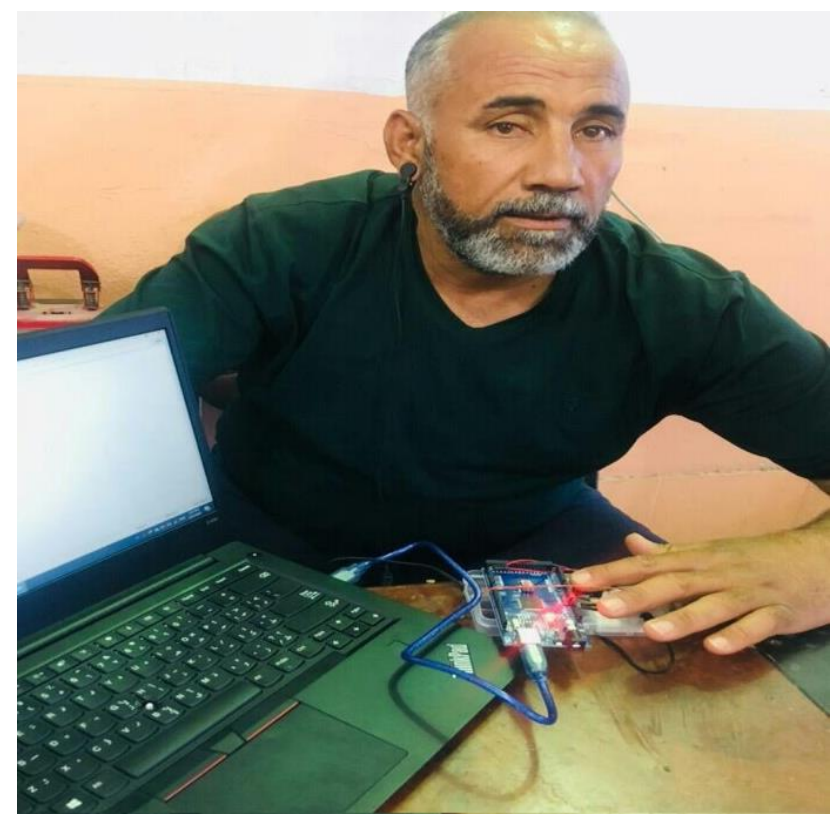

Figure 11. Experimental environment

\subsection{Data display in plotter}

In the Fig 11 graph of the blue color represents information of heart rate sensor signal and the second graph contains the green heart rate sensor signal repeats every 15 seconds and the red skin temperature sensor signal repeats every two seconds depending on the delay specified in the program. Whereas, the $\mathrm{x}$ axis represents the time and the $y$ axis represents the amplitude.
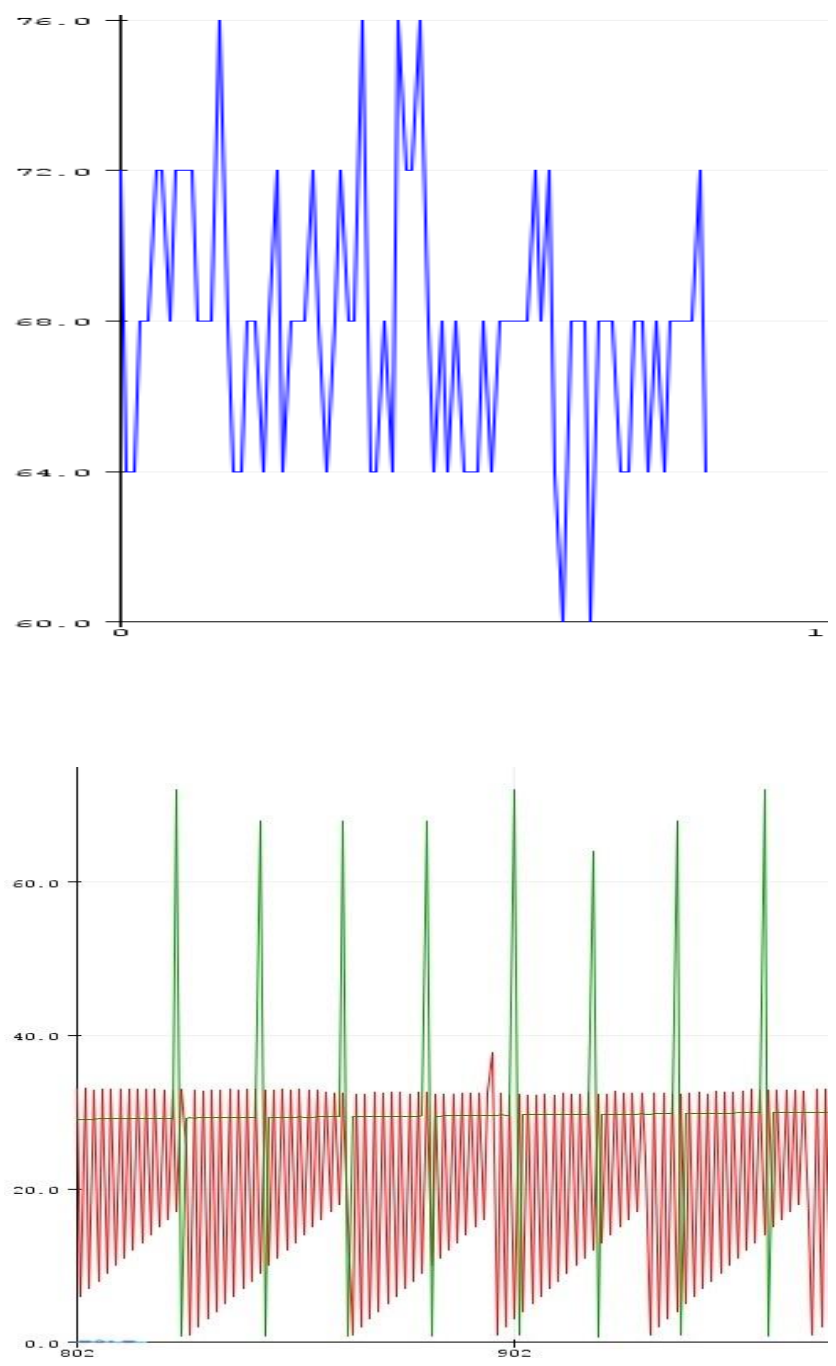

Figure 12. Screenshot: sensing signals in plotter

In addition, the system gives room temperature in order to prevent the influence of temperature changes on the health condition of the observer.

\subsection{Testing and findings health care unit}

The developed Patient Health Monitoring System is checked to use different people from normal to uncommon states of health. The different tests and results give minimum error rate and the findings are described below.

Through the tests that I conducted on several people, especially the elderly and shown in the 
photos below. I have noticed when the vital signs of the body (heartbeat and skin temperature) are within the normal range, the smart system gives a low risk ratio, unlike if one of the vital signs out of the normal range, the smart system will give a risk ratio that is appropriate to these data, so caregivers will be able to know how dangerous the observed patient is, with ease and speed, to take the necessary measures. The down in heart beat is more dangerous than the high heartbeat.

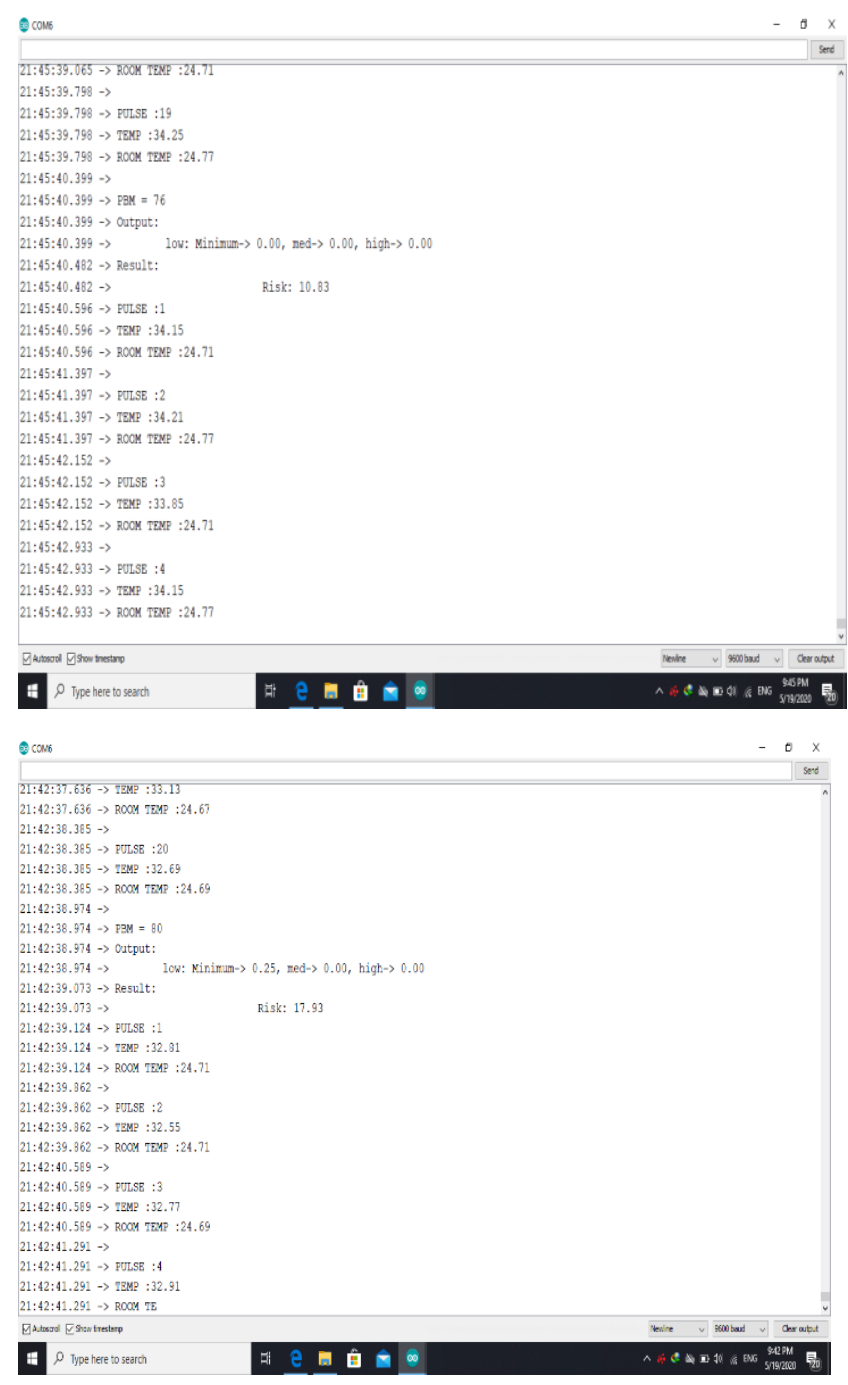

Figure 12.a Results for normal people

In fig 12.a Results for two healthy people, their skin temperature ranged between (32 to 35), and the heartbeat ranged between (75 to 85$)$, and all of these results were within the normal range, so their degree of risk was relatively low, less than $(20 \%)$.

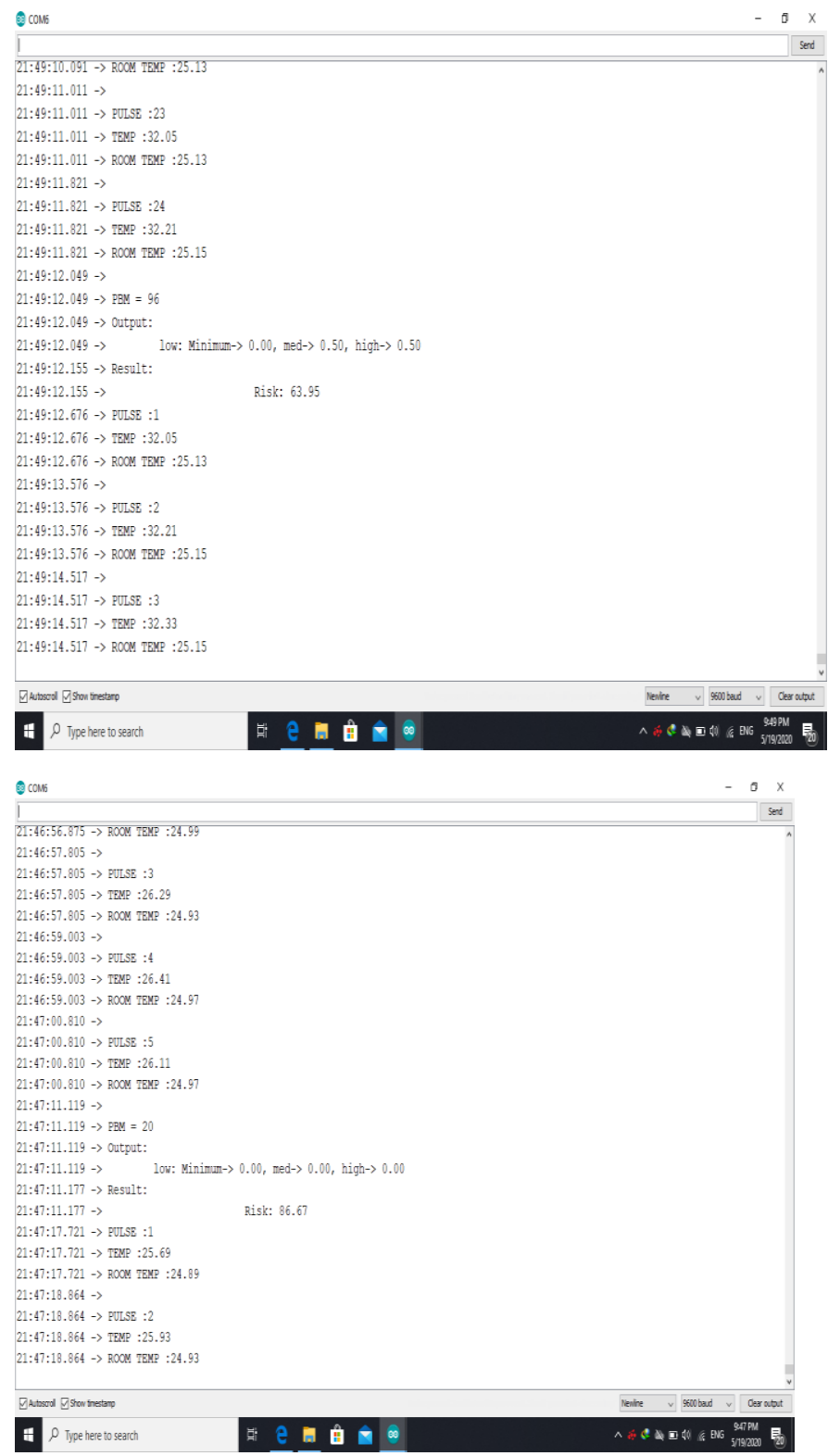

Figure 12.b Results for abnormal people

In fig 12.b Results for two people. The first is a normal skin temperature, but his heartbeat is slightly higher than normal, so the risk ratio has risen to $(63 \%)$. As for the second person, his skin temperature was relatively low, and his heartbeats were very low, and this is a dangerous condition, so the risk rate was high (86\%). 
Table 2. The result for four cases

\begin{tabular}{|l|c|c|c|}
\hline & Skin temp & Heart rate & Degree of risk \\
\hline Person 1 & 34.25 & 76 & 10.83 \\
\hline Person 2 & 32.69 & 80 & 17.93 \\
\hline Person 3 & 32.21 & 96 & 63.95 \\
\hline Person 4 & 26.11 & 20 & 86.67 \\
\hline
\end{tabular}

\section{5. conclution}

The Use of an intelligent system (fuzzy logic) technology in healthcare can reduce the need for hospitals, and thus fewer deaths, especially among the elderly. This paper proposes and explains a very easy-to-use device and uses the fuzzy logic system found in the Arduino libraries to analyze the recorded values from the sensors, and then gives the risks ratio directly. It found through the experiment that using this system provides good, reliable, quick and accurate, even though some of the components used are not original. As for using an original and well-made component company, this system can be used throughout the day for monitoring without any problems, as it is easy to use and suitable for the elderly.

It is easy in the future to upload information obtained from the device to the platform through the Internet of Things so that information can be accessed for diagnosis and intensive care in all hospitals.

\section{Conflict of interest}

The publication of this article causes no conflict of interest.

\section{Reference}

1. Hussain, Aamir, et al. "Personal home healthcare system for the cardiac patient of smart city using fuzzy logic." Journal of
Advances in Information Technology Vol 7.1 (2016).

2. Mahfouf, Mahdi, Maysam F. Abbod, and Derek A. Linkens. "A survey of fuzzy logic monitoring and control utilization in medicine." Artificial intelligence in medicine 21.1-3 (2001): 27-42.

3. Garibaldi, Jonathan M., et al. "Incorporation of expert variability into breast cancer treatment recommendation in designing clinical protocol guided fuzzy rule system models." Journal of biomedical informatics 45.3 (2012): 447-459.

4. Suryawanshi, Chandani, and Bhakti Kurhade. "Healthcare Monitoring System Based on Pulse Sensor." Ijsr. Net 4.4 (2013): 29462949.

5. Tiwari, Niyati, et al. "Simulation, feature extraction and disorder detection (using fuzzy logic) of uterine contractions." 2014 6th International Conference of Soft Computing and Pattern Recognition (SoCPaR). IEEE, 2014.

6. Abbas, Basim Khudair. "Simulation Models of Emergency Department in Hospital." Journal of Engineering and Sustainable Development 18.2 (2014): 172-179.

7. Pandey, Sanjib Raj, Jixin Ma, and Choi-Hong Lai. "Development of decision support system for the diagnosis of arthritis pain for rheumatic fever patients: based on the fuzzy approach." Journal of Algorithms \& Computational Technology 9.3 (2015): 265-290.

8. Al-Dmour, Jumanah A., et al. "A fuzzy logicbased warning system for patiAl-Dmour, Jumanah A., et al. "A fuzzy logic-based warning system for patient's classification." Health informatics journal 25.3 (2019): 10041024. ents classification." Health informatics journal 25.3 (2019): 1004-1024.

9. ROSS, TIMOTHY J. "Fuzzy Logic with Engineering Applications." (1997). 
10. Zadeh, Lotfi A. "Making computers think like people [fuzzy set theory]." IEEE spectrum 21.8 (1984): 26-32.

11. Al-Dmour, Jumana. Fuzzy Logic Based Patients' Monitoring System. Diss. 2013.

12. Al-awad, Nassir Ahmed. "FUZZY PROPORTIONAL-DERIVATIVE SLIDING MODE CONTROL WITH MINIMAL RULES FOR DISK READ SYSTEM." Journal of Engineering and Sustainable Development 21.3 (2017): 12-23.

13. K. Vani and R. R. Neeralagi, "IoT based health monitoring using fuzzy logic," International Journal of Computational Intelligence Research, vol. 13, pp. 2419-2429, 2017.

14. A.H. Mary, Tolgay Kara, and A.H. Miry, "Inverse kinematics solution for robotic manipulators based on fuzzy logic and PD control," Al-Sadiq International Conference on Multidisciplinary in IT and Communication Techniques Science and Applications, IEEE, pp. 1-6, 2016.

15. M A. A.li, A. Hussein Miry, T.M. Salman, Implementation of Intelligent Industrial Controller Based on Fuzzy Logic and PLC ", AL-QADISIYAH JOURNALFORENGINEERING SCIENCES 13 (2020) 054-059

16. R. S. Krishnan, E. G. Julie, Y. H. Robinson, S. Raja, R. Kumar, and P. H. Thong, "Fuzzy Logic based Smart Irrigation System using Internet of Things," Journal of Cleaner Production, vol. 252, p. 119902, 2020.

17. Surawicz, Borys, C. Pratap Reddy, and Eric N. Prystowsky, eds. Tachycardias. Vol. 28. Springer Science \& Business Media, 2012.

18. Banzi, Massimo, and Michael Shiloh. Getting started with Arduino: the open source electronics prototyping platform. Maker Media, Inc., 2014.
19. A. H. Miry, G. A. Aramice ,"Water monitoring and analytic based ThingSpeak ", International Journal of Electrical and Computer Engineering (IJECE) Vol. 10, No. 4, August 2020, pp:3588 3595

20. Valldorf, Jürgen, and Wolfgang Gessner, eds. Advanced microsystems for automotive applications 2005. Berlin: Springer, 2005.

21. Farin, Nusrat J., S. M. A. Sharif, and Iftekharul Mobin. "An intelligent sensorbased system for real time heart rate monitoring (HRM)." (2016). 